\title{
O método de aprendizagem baseada em problemas na disciplina de Radiologia Odontológica
}

\author{
Neiandro dos Santos Galvão*; Sergio Lins de-Azevedo-Vaz**; Matheus Lima Oliveira*** \\ * Doutorando, Programa de Pós-Graduação em Radiologia \\ Odontológica da Faculdade de Odontologia de Piracicaba, \\ UNICAMP \\ ** Professor Adjunto, Departamento de Clínica Odontológica do \\ Centro de Ciências da Saúde da Universidade Federal do Espírito \\ Santo \\ *** Professor Doutor, Área de Radiologia Odontológica, Faculdade de \\ Odontologia de Piracicaba, UNICAMP
}

Recebido em 24/10/2016. Aprovado em 26/11/2016.

\begin{abstract}
RESUMO
Na presente pesquisa propôs-se avaliar a opinião de estudantes de Odontologia quanto à experiência vivenciada mediante aplicação da aprendizagem baseada em problemas (Problem-Based Learning PBL) na disciplina de Radiologia Odontológica. A amostra foi composta por 138 estudantes de uma instituição de ensino privada, sendo a maior parte do gênero feminino $(75,20 \%, \mathrm{n}=104)$. Houve predomínio da faixa etária de 19 a 20 anos $(39,59 \%, \mathrm{n}=56)$, de estudantes oriundos de escolas públicas $(62,19 \%, \mathrm{n}=86)$ e com renda familiar mensal entre $\mathrm{R} \$ 4.501,00$ e $\mathrm{R} \$ 9.500,00(22,79 \%$; $\mathrm{n}$ =32). Quando questionados sobre o método PBL, um total de $127(92,00 \%)$ considerava válida a experiência com novos métodos de ensino-aprendizagem e 129 (93,53\%) não o conheciam. Ainda, $123(89,05 \%)$ acreditavam que a timidez pode atrapalhar o estudante no método PBL e $113(82,14 \%)$ concordavam que o professor tem uma participação discreta nessa metodologia de ensino. Após a avaliação, 108 estudantes $(78,49 \%)$ não se mostraram satisfeitos com a nota atribuída e 105 (76,38\%) não gostariam de estudar em uma Instituição na qual todas as disciplinas utilizassem o PBL. Concluiu-se que os estudantes participantes deste estudo apresentaram dificuldades com o método PBL e não estiveram plenamente satisfeitos quando participaram de atividades nesse formato. Acredita-se que esses achados possam ser explicados pelas peculiaridades de abordagem dos conteúdos e critérios de avaliação que envolvem habilidades e competências, indicadores que vão além do conhecimento cognitivo e com os quais os estudantes não estão habituados.

Descritores: Aprendizagem Baseada em Problemas. Odontologia. Radiologia. Ensino.
\end{abstract}

\section{INTRODUÇÃO}

No atual contexto social, no qual os meios de comunicação estão potencializados pelo avanço das novas tecnologias e pela percepção do mundo como uma rede de relações dinâmicas e em constante transformação, tem-se discutido a necessidade de urgentes mudanças nas instituições de ensino visando, entre outros aspectos, à reconstrução de seu papel social ${ }^{1}$.

As metodologias ativas de aprendizagem 
vêm ganhando espaço por estarem alicerçadas em um princípio teórico significativo: a autonomia. A educação contemporânea deve considerar um discente capaz de autogerenciar ou autogovernar seu processo de formação independente da sua área de formação ${ }^{2}$.

$\mathrm{O}$ método da aprendizagem baseada em problemas (do inglês, problem-based learning PBL) é uma metodologia de ensino na qual pequenos grupos de estudantes, orientados por tutores, desenvolvem-se a partir de objetivos de aprendizagem com base em problematizações reais e virtuais. Em seguida, após estudarem de forma independente, se reúnem e discutem o problema proposto com o objetivo de resolver o caso $^{3,4}$.

O PBL foi introduzido pela primeira vez na educação odontológica na Faculdade de Odontologia de Malmö, na Suécia, em 1990. A implementação do PBL no ensino odontológico aumentou gradualmente durante os anos $1990 \mathrm{e}$ 2000, de modo que em 2009 quase metade das faculdades de Odontologia dos Estados Unidos e do Canadá havia adotado esse método pedagógico ${ }^{5,6,7}$. Algumas instituições brasileiras já adotam o PBL nos cursos de Medicina, como a Universidade Estadual de Londrina (UEL), Faculdade de Medicina de Marília (FAMENA), Universidade de São Francisco (USF) e Pontifícia Universidade Católica de São Paulo (PUC-SP). ${ }^{3}$

Apesar de ser uma metodologia já consolidada na literatura, o seu uso no ensino odontológico brasileiro é muito discreto e suas experiências são pouco documentadas e discutidas. Com base nos resultados positivos obtidos com o uso da metodologia PBL na disciplina de Radiologia Odontológica nas Universidades de Taiwan e, recentemente, da Espanha, e considerando a importância do contexto sociocultural no processo de ensino- aprendizagem, o objetivo no presente estudo foi avaliar as contribuições do uso desse método no ensino da Radiologia Odontológica em uma realidade brasileira sob a perspectiva dos estudantes $^{8,9}$.

\section{MATERIAL E MÉTODOS}

O projeto desta pesquisa foi aprovado pelo Comitê de Ética em Pesquisa com seres humanos local, sob o protocolo 1.117.143/2015.

Tratou-se de um estudo observacional com delineamento transversal e abordagem quantitativa descritiva. A população estudada foi composta por estudantes do Curso de Odontologia de uma Instituição de Ensino Superior privada localizada na Região Norte do Brasil. Os critérios de inclusão foram: estar regularmente matriculado na disciplina de Radiologia Odontológica e ter participado assiduamente das atividades didáticas no formato PBL desenvolvidas na disciplina ao longo do semestre.

A amostra foi dividida em grupos de 8 a 10 estudantes, seguindo as recomendações e as etapas clássicas do método $\mathrm{PBL}^{3,4}$ com o objetivo de solucionar os problemas levantados em cada encontro. O método PBL contém as seguintes etapas: apresentação do problema (leitura pelo grupo); definição e resumo do problema, com identificação de áreas/pontos relevantes; análise do problema utilizando os conhecimentos prévios (tempestade de idéias - brainstorm); desenvolvimento de hipóteses para explicar o problema e identificação de lacunas de conhecimento; definição dos objetivos de aprendizagem e identificação dos recursos de aprendizagem apropriados; busca de informação e estudo individual; compartilhamento da informação obtida e aplicação na compreensão do problema e avaliação do trabalho do grupo e dos seus membros. ${ }^{3,4}$ 
Concomitantemente às discussões, os estudantes foram avaliados por meio de ficha preenchida pelo tutor que incluía os seguintes critérios: habilidade de identificar o problema, conhecimentos prévios, capacidade de gerar hipóteses, sintetizar e expor ideias de forma clara e organizada, além da pontualidade, frequência e relação interpessoal. Um profissional com pósgraduação em metodologia do ensino superior, mestrado em Radiologia Odontológica e com experiência docente em graduação de seis anos exerceu a função de tutor do método PBL.

Um questionário autoadministrado, estruturado, com respostas objetivas do tipo "Sim" ou "Não" e adaptado de Chen et al. (2001) foi aplicado. O questionário continha questões abordando as percepções do estudante quanto aos métodos de ensino e de avaliação, além de suas opiniões acerca das atividades no formato PBL desenvolvidas na disciplina Radiologia Odontológica. O estudante tinha liberdade de tempo e privacidade para responder o questionário, não sendo identificado em nenhuma etapa da pesquisa.

Ao final da aplicação do instrumento, os dados coletados foram tabulados no programa Microsoft Excel 2016 e tratados por estatística descritiva (frequência simples e percentual).

\section{RESULTADOS}

Todos os estudantes do universo amostral aceitaram participar da pesquisa. Dessa forma, a taxa global de resposta foi de $100 \%$, resultando em um número amostral de 138 participantes. A maior parte da amostra $(75,20 \%)$ constituía-se de acadêmicos do sexo feminino $(\mathrm{n}=104)$ com idade entre 19 e 20 anos $(39,59 \% ; \mathrm{n}=56)$, com formação anterior em escola pública $(62,19 \%$; n = 86) e renda familiar entre $\mathrm{R} \$ 4.501,00$ e $\mathrm{R} \$$ $9.500,00(22,79 \% ; \mathrm{n}=32)($ tabela 1$)$.

Na tabela 2 são demonstrados os resultados obtidos com a aplicação do instrumento utilizado neste estudo. Um total de 106 estudantes (76,96\%) acreditava que o método de ensinoaprendizagem adotado pelo professor pudesse interferir no aprendizado; 127 estudantes $(92,00 \%)$ consideravam a experiência com novos métodos de ensino-aprendizagem válida e 129 estudantes $(93,53 \%)$ não conheciam o método PBL.

Ainda, 123 estudantes $\quad(89,05 \%)$ acreditavam que a timidez poderia atrapalhar o estudante no método PBL e 113 (82,14\%) concordavam que o professor tem uma participação discreta nessa metodologia de ensino. Após a avaliação, 108 estudantes $(78,49 \%)$ não se mostraram satisfeitos com a nota recebida e 105 estudantes $(76,38 \%)$ não gostariam de estudar em uma Instituição na qual todas as disciplinas utilizassem o método PBL.

\section{DISCUSSÃO}

O levantamento sociodemográfico mostrase importante, nos estudos relacionados ao processo de ensino-aprendizagem, uma vez que caracteriza o grupo amostral e pode estar relacionado com os resultados encontrados em estudos sobre o processo de ensinoaprendizagem. Neste estudo, além da predominância do sexo feminino e da faixa etária entre 19 e 20 anos, a maioria dos estudantes eram oriundos do ensino público e apresentavam renda familiar mensal entre $\mathrm{R} \$ 4.501,00$ e $\mathrm{R} \$ 9.500,00$.

Os estudantes participantes deste estudo concordaram que o método de ensinoaprendizagem adotado pelo professor interfere em seu aprendizado e consideravam válida a experiência com novos métodos. Esses resultados nos levam a pensar que os estudantes acreditam que existam métodos mais eficientes que outros e estão dispostos a experimentar novas metodologias em sala de aula. 
Embora o método tradicional de ensino atinja um maior número de estudantes e permita a transmissão de mais informações em menor tempo, atualmente, os educadores têm recomendado sua substituição por modelos mais dinâmicos que objetivam desenvolver o pensamento crítico, a aprendizagem autônoma e a resolução de problemas ${ }^{4}$. Essa recomendação deve-se aos requisitos fundamentais exigidos aos profissionais da saúde, tais como o de avaliar, sistematizar e decidir as condutas mais adequadas, baseadas em evidências científicas ${ }^{10}$.

\begin{tabular}{ccc} 
Tabela 1. Caracterização sociodemográfica da amostra $-\mathrm{n}(\%)$ \\
\hline \multirow{2}{*}{ Sexo } & Masculino & $34(24,80)$ \\
& Feminino & $104(75,20)$ \\
\hline \multirow{4}{*}{ Idade (anos) } & $17-18$ & $35(25,61)$ \\
& $19-20$ & $56(39,59)$ \\
Formação & $21-22$ & $20(14,57)$ \\
& $23-24$ & $9(6,82)$ \\
& Escola pública & $86(62,19)$ \\
& Escola privada & $42(30,49)$ \\
& Outros* & $10(7,32)$ \\
\hline \multirow{4}{*}{ Renda familiar } & De 501 a 1.500 reais & $6(4,68)$ \\
& De 1.501 a 2.500 reais & $18(13,68)$ \\
& De 2.501 a 4.500 reais & $31(22,67)$ \\
& De 4.501 a 9.500 reais & $32(22,79)$ \\
& Mais de 9.500 reais & $25(17,73)$ \\
& Não sabe & $26(18,45)$ \\
\hline
\end{tabular}

*Outros: formados por supletivo ou bolsistas de escolas privadas.

Contudo, quando os estudantes participantes deste estudo foram questionados sobre as características dos métodos tradicional e PBL, observou-se uma considerável preferência ao método tradicional. Os estudantes consideraram, por exemplo, que no PBL o professor tem uma participação discreta, que teriam melhor aproveitamento em aulas no formato tradicional e que não gostariam de estudar em uma instituição onde todas as disciplinas fossem no formato PBL. Esses dados podem estar relacionados à pouca experiência dos estudantes com métodos ativos de aprendizagem como o PBL. A literatura científica levantada ${ }^{8,9}$ apresenta dois estudos que tratam do uso do método PBL em Radiologia Odontológica. Eles sinalizam resultados satisfatórios na correlação do método com a 
disciplina. Entretanto, vale considerar o contexto sociocultural e individual dos estudantes de diferentes países. No Brasil, o ensino tradicional ainda é o mais empregado e difundido método de ensino, tanto no ensino infantil quanto na formação acadêmica ${ }^{11-15}$. Essa realidade pode ocasionar certo desconforto comum a qualquer processo de mudança e adaptação para o estudante em sala de aula, sendo um dos motivos pelos quais os estudantes brasileiros apresentaram resultados diferentes em comparação com os estudos anteriores ${ }^{8,9}$.

Tabela 2. Avaliação do método PBL pelos estudantes $(\mathrm{n}=138)$.

\begin{tabular}{lcc}
\multicolumn{1}{c}{ QUESTÕES } & SIM & NÃO \\
& n(\%) & n (\%) \\
\hline $\begin{array}{l}\text { 1- Você acredita que o método de ensino-aprendizagem } \\
\text { adotado pelo professor pode interferir no aprendizado do } \\
\text { aluno? }\end{array}$ & $106(76,96)$ & $32(23,04)$ \\
\hline $\begin{array}{l}\text { 2- Você considera válida a experiência com novos métodos de } \\
\text { ensino-aprendizagem? }\end{array}$ & $127(92,00)$ & $11(8,00)$ \\
\hline $\begin{array}{l}\text { 3- Você tinha conhecimento do método PBL antes dessa } \\
\text { experiência? }\end{array}$ & $9(6,46)$ & $129(93,53)$ \\
\hline $\begin{array}{l}\text { 4- Você concorda que no método PBL o professor tem uma } \\
\text { participação discreta? }\end{array}$ & $113(82,14)$ & $25(17,86)$ \\
\hline $\begin{array}{l}\text { 5- Você considera a participação discreta do professor } \\
\text { prejudicial no processo de ensino-aprendizagem? }\end{array}$ & $87(63,49)$ & $51(36,50)$ \\
\hline $\begin{array}{l}\text { 6- Você acredita que estas aulas seriam melhor aproveitadas no } \\
\text { formato tradicional? }\end{array}$ & $93(67,76)$ & $45(32,24)$ \\
\hline $\begin{array}{l}\text { 7- Você acredita que aprenderia melhor a matéria no formato } \\
\text { de aulas tradicionais? }\end{array}$ & $89(64,86)$ & $49(35,13)$ \\
\hline $\begin{array}{l}\text { 8- Você receberia bem a ideia de estudar em uma Instituição } \\
\text { onde todas as disciplinas fossem no formato PBL? }\end{array}$ & $33(23,62)$ & $105(76,38)$ \\
\hline $\begin{array}{l}\text { 9- Você acredita que uma avaliação diária de desempenho } \\
\text { possa substituir satisfatoriamente uma avaliação tradicional? }\end{array}$ & $96(69,50)$ & $42(30,50)$ \\
\hline $\begin{array}{l}\text { 10- Você concorda com os critérios de avaliação do método } \\
\text { PBL? }\end{array}$ & $75(53,70)$ & $63(46,30)$ \\
\hline $\begin{array}{l}\text { 11- Você sentiu-se constrangido em participar das discussões } \\
\text { em grupo? }\end{array}$ & $40(28,92)$ & $98(71,07)$ \\
\hline $\begin{array}{l}\text { 12- Você considera que o aluno tímido é prejudicado no método } \\
\text { PBL? }\end{array}$ & $123(89,05)$ & $15(10,94)$ \\
\hline $\begin{array}{l}\text { 13- Você apresenta dificuldades para estudar sozinho? } \\
\text { 14- Sua nota na PBL correspondeu às suas expectativas? }\end{array}$ & $58(41,94)$ & $80(58,06)$ \\
\hline \begin{tabular}{l} 
15- Você se sente intimidado para falar em público? \\
\hline
\end{tabular} & $81(58,51)$ & $108(78,49)$ \\
\hline
\end{tabular}

Outro fator que não deve ser desprezado quando se interpreta os resultados deste estudo é o elevado número de estudantes insatisfeitos com a pontuação final obtida com o processo de avaliação realizado no PBL, o que pode ter contribuído para a rejeição ao método. Apesar de acreditarem que a avaliação diária de desempenho possa substituir satisfatoriamente 
uma prova formal e concordarem com os critérios utilizados na avaliação do PBL, o fato de se exigir mais que o conhecimento cognitivo gerou insatisfação e pode ter repercutido diretamente na avaliação dos estudantes sobre o método.

Os estudantes concordaram que o acadêmico tímido pode ser prejudicado, considerando que para ser avaliado, ele deve participar ativamente das discussões em grupo. Todavia, a habilidade de se expressar verbalmente de forma clara e organizada, assim como outras desenvolvidas no método PBL, é uma das características requeridas do cirurgiãodentista durante o exercício da profissão e deve ser desenvolvida no ambiente acadêmico. ${ }^{16}$

Acredita-se que esses achados possam ser explicados pelas peculiaridades de abordagem dos conteúdos e critérios de avaliação no método PBL que envolvem habilidades e competências, indicadores que ultrapassam o conhecimento cognitivo e com os quais os estudantes não estão habituados.

A comparação científica entre métodos de ensino-aprendizagem é complexa e apresenta limitações inerentes ao tema. No entanto, o desenvolvimento de estudos do tipo casocontrole com grupos pareados, prospectivos e testes que avaliem habilidades e competências podem vir a contribuir ainda mais com resultados sobre o uso do método PBL em Radiologia Odontológica. Tais estudos incentivariam a reflexão dos docentes da área sobre o uso das metodologias ativas nas instituições de ensino. Pesquisas recentes ${ }^{17}$ apontam para um modelo curricular integrado e que reúna diferentes métodos de ensino-aprendizagem visando atingir melhores desempenhos dos estudantes, considerando os perfis socioculturais e psicológicos que estão diretamente relacionados com a aprendizagem.

\section{CONCLUSÕES}

Concluiu-se que os estudantes participantes deste estudo apresentaram dificuldades com o método PBL e não estiveram plenamente satisfeitos quando participaram de atividades nesse formato, contudo, questões socioculturais relacionadas ao processo de ensinoaprendizagem devem ser consideradas em estudos posteriores.

\section{ABSTRACT \\ The problem-based learning method in Dental Radiology}

The aim of this study was to evaluate the opinion of dental students about their experience with the problem-based learning (PBL) method in the Dental Radiology discipline. A total of 138 students participated in this study, out of which 104 were females (75.20\%). The sample was mostly composed of students between 19-20 years old $(39.59 \%, \mathrm{n}=56)$ from public schools $(62.19 \%, \mathrm{n}=$ 86) and with monthly income between $\mathrm{R} \$ 4,501.00$ and $\mathrm{R} \$ 9,500.00(22.79 \%, \mathrm{n}=32)$. When they were asked about the PBL method, 127 (92.00\%) considered positive the experience with new teaching-learning methods and 129 (93.53\%) were not aware of the PBL method. The results also showed that 123 students $(89.05 \%)$ believed that shyness can negatively affect learning in the PBL method and $113(82.14 \%)$ agreed that the professor has a discrete role. After the exams, 108 students $(78.49 \%)$ were not satisfied with their grade and 105 (76.38\%) would not appreciate to study in an institution in which the PBL was applied in all disciplines. As a conclusion, the students who participated in this study had difficulties with the PBL method and were not fully satisfied when participating in activities with such a format. These findings can be explained by the particular approach of the contents in the PBL method, further the evaluation criteria involving skills and competencies. These are indicators that go beyond cognitive knowledge and which the students are not used to consider.

Descriptors: Problem-Based Learning. Dentistry. Radiology. Teaching. 


\section{REFERÊNCIAS}

1. Mitre SM, Siqueira-Batista R, Girardi MJM, Morais-Pinto NM, Meirelles CAB, PintoPorto C, et al. Metodologias ativas de ensino-aprendizagem na formação profissional em saúde: debates atuais. Ciênc Saúde Coletiva. 2008;13(Supl 2):S2133-44.

2. Freire P. Pedagogia da autonomia: saberes necessários à prática educativa. 33a ed. São Paulo: Paz e Terra; 2006.

3. Prado AS. Alternativas pedagógicas em uma disciplina de mestrado de um curso de odontologia. Um projeto exploratório [dissertação]. São Paulo: Pontifícia Universidade Católica de São Paulo; 2005.

4. Davis BG. Tools for teaching. New York: John Wiley \& Sons, 2009.

5. Rohlin M, Petersson K, Svensater G. The Malmo model: a problem-based learning curriculum in undergraduate dental education. Eur J Dent Educ. 1998;2(3):10314.

6. Kassebaum DK, Hendricson WD, Taft T, Haden NK. The dental curriculum at North American dental institutions in 2002-03: a survey of current structure, recent innovations, and planned changes. J Dent Educ. 2004;68(9):914-31.

7. Haden NK, Hendricson WD, Kassebaum DK, et al. Curriculum change in dental education, 2003-09. J Dent Educ. 2010;74(5):539-57.

8. Chen SK, Chang HF, Chiang CP. Group learning factors in a problem-based course in oral radiology. Dentomaxillofac Radiol. 2001;30:84-7.

9. Palmares-Casado T. Enseñanza de la radiología y medicina física en el grado en odontología a través del aprendizaje basado en problemas. FEM: Rev Fund Educ Méd. 2014;17(4):221-8.

10. Arias A, Scott R, Peters OA, McClain E, Gluskin AH. Educational outcomes of small-group discussion versus traditional lecture format in dental students' learning and skills acquisition. J Dent Educ. 2016; 80(4):459-65.
11. Saliba NA, Moimaz SAS, Chiaratto RA, Tiano AVP. A utilização da metodologia PBL em Odontologia: descortinando novas possibilidades ao processo ensinoaprendizagem. Rev Odonto Ciênc. 2008; 23(4):392-6.

12. Noro LRA, de Santana Farias-Santos BC, Sette-de-Souza PH, Pinheiro IAG, Borges R EA, Ferreira LM. O professor (ainda) no centro do processo ensino-aprendizagem em Odontologia. Rev ABENO. 2015;15(1):211.

13. de Souza FN, Barros RN, de Almeida CC, de França M, Hayassy A. Comparação dos métodos tradicional e ativo de educação no aprendizado de um tema de Oclusão Dentária. Rev ABENO. 2016;15(4):60-6.

14. Rocha JS, Dias GF, Campanha NH, Baldani MH. O uso da aprendizagem baseada em problemas na Odontologia: uma revisão crítica da literatura. Rev ABENO. 2016; 16(1):25-38.

15. Borges RN, de Melo M, Freitas GC, de Barcelos BA, Arantes BM. Utilização da metodologia "problem based learning" na disciplina de oclusão, na FO/UFG. ROBRAC.2015;24(71):174-7.

16. Brasil. Ministério da Educação. Resolução $\mathrm{n}^{\circ}$ CNE/CES 3/2002 de 19 de fevereiro de 2002. Institui as Diretrizes Curriculares Nacionais dos Cursos de Farmácia e Odontologia. Diário Oficial, Brasília, 04 mar 2002, seção 1, p. 10.

17. Elangovan S, Venugopalan SR, Srinivasan S, Karimbux NY, Weistroffer P \& Allareddy V. Integration of Basic-Clinical Sciences, PBL, CBL, and IPE in US Dental Schools' curricula and a proposed integrated curriculum model for the future. J Dent Educ. 2016; 80(3):281-90.

Correspondência para:

Neiandro dos Santos Galvão e-mail: neiandrogalvao@gmail.com

Av. Limeira, 901, Vila Rezende

13414-903 - Piracicaba/SP 